\title{
Diagnosis | Vesical calculus-induced obstructive dystocia
}

We made a final diagnosis of vesical calculus-induced obstructive dystocia. The bladder stone effectively blocked the vaginal canal causing dystocia. In humans, complications related to bladder calculi are usually associated with infection; however, fistula formation, stillbirths, and obstruction of labor have been reported ${ }^{1,2}$. Only 10 human cases of cystic calculi formation leading to dystocia were documented in the last century ${ }^{1,2}$.

To our knowledge, there have been no reported cases of cystic calculi formation leading to labor obstruction in veterinary medicine. Sprague-Dawley (as well as Wistar) rats are highly susceptible to renal disease ${ }^{3,4}$ and uremic neuropathy is one of the major causes of mortality in these rats ${ }^{5}$. Renal problems and kidney stone formation have been observed since the 1960s in rats. Most stones were composed of calcium oxalate or other calcium salts. So frequent was this problem that the American Institute of Nutrition (AIN) established a semipurified rodent (AIN-76A) diet that would meet the nutritional requirements of rodents and provide a consistent control diet for nutritional and toxicological studies. However, high incidences of nephrocalcinosis were still reported in female Sprague-Dawley, Wistar, Zucker, and Fischer 344 rats. Male rats of all strains appeared to be less susceptible to the development of this type of lesion. The AIN subsequently reformulated the AIN-76A diet to become the AIN-93 diet that had a revised calcium to phosphorus molar ratio to further reduce the incidence of nephrocalcinosis in female rats ${ }^{6}$. The rats in our study were fed the AIN-93 diet ad libitum. It was reported, however, that the manufacturer of the AIN-93 diet reputably altered its source of choline bitartrate, resulting in a diet that contained approximately seven times the level of choline that is recommended in the AIN formula. The feeding of this altered, purified diet was linked to an increased incidence of kidney and bladder stones, renal failure, and death of numerous rats from various international institutions ${ }^{7,8}$. Data on some stones indicated that they were not calcium rich and may contain tricholine citrate ${ }^{7}$. Chemical analysis of the calculus we found in this rat showed an uncommon magnesium ammonium phosphate composition. In addition to the dam in this case report, a total of 18 out of 35 dams in our study also developed urinary calculi.

The elevated BUN and creatinine we saw in the clinical chemistry results may have been related to obstructive renal failure from the bladder stone or renal failure independent of the stone that was related to the diet. It is possible that the Sprague Dawley's genetic predisposition combined with environmental factors such as the AIN-93 diet increased the incidence of calculi formation in the rats in our study. These factors may also have contributed to the development of a vesical calculus large enough in size to interfere with normal labor and delivery in a Sprague-Dawley rat.

\section{ABSTRACT}

Vesical calculus is a rare cause of dystocia. Only 10 human cases were documented in the last century. There have been no reported incidences of cystic calculi formation leading to dystocia in veterinary medicine. We report a dystocia in a day-23 timed pregnant Sprague-Dawley rat caused by a magnesium ammonium phosphate urinary bladder calculus obstructing the vaginal canal.

\section{ACKNOWLEDGMENTS}

The authors thank Drs. Xiao-Juan Wang and Mark Cesta for their reviews of this case report.

1. Cope, E.C. Obstructed labour due to vesical calculus. J. Obst. Gynaecol. Br. Commonw. 68, 476-478 (1961).

2. Egwuatu, V.E. Bladder calculus with pregnancy. J. Urol. 123(6), 954-955 (1980).

3. Gray, J.E. in Urinary System (eds. Jones, T.C., Mohr, U \& Hunt, R.D.) 174-179 (SpringerVerlag, New York, 1986).

4. Montgomery, C.A. Jr. \& Seely, J.C. in Pathology of the Fischer Rat (eds. Boorman, G.A., Eustis, S.L., Elwell, M.R., Montgomery, C.A. Jr. \& MacKenzie, W.F.) 132-134 (Academic Press, New York, 1990).

5. Haseman, J.K., Eustis, S.L. \& Ward, J.M. in ILSI Monograph on Pathology of Aging Animals (eds. Mohr, U., Capen, C. \& Dungworth, D.) 629-638 (Springer-Verlag, New York, 1993).

6. Reeves, P.G., Nielsen, F.H. \& Fahey, G.C. Jr. AIN93 purified diets for laboratory rodents: final report of the American Institute of Nutrition ad hoc writing committee on the reformulation of the AIN-76A rodent diet. J. Nutr. 123(11), 1939-1951 (1993).

7. Klurfeld, D.M. Kidney and bladder stones in rodents fed purified diets. J. Nutr. 132(12), 3784 (2002).

8. Day, J.J., Reed, M.N. \& Newland, M.C. Neuromotor deficits and mercury concentrations in rats exposed to methyl mercury and fish oil. Neurotoxicol. Teratol. 27(4), 629-641 (2005).

LAB ANIMAL welcomes reader contributions to "What's Your Diagnosis" in case history/diagnosis format. Submissions should include two to five illustrations or photos. Please email manuscripts to editors@labanimal.com. Selections are made on the basis of relevance and interest to readers. Please refer to the Guide to Authors for information on submission of digital figures. 